Mathematical Modelling And Analysis

Volume 21 Number 1, January 2016, 35-46

http://dx.doi.org/10.3846/13926292.2016.1128990

(c) Vilnius Gediminas Technical University, 2016
Publisher: Taylor\&Francis and VGTU

http://www.tandfonline.com/TMMA

ISSN: $1392-6292$

eISSN: $1648-3510$

\title{
Universality Theorems for Some Composite Functions
}

\section{Kęstutis Janulis ${ }^{a}$, Donatas Jurgaitis ${ }^{b}$, Antanas Laurinčikas ${ }^{a}$ and Renata Macaitiené ${ }^{c}$}

\author{
${ }^{a}$ Faculty of Mathematics and Informatics, Vilnius University \\ Naugarduko str. 24, LT-03225 Vilnius, Lithuania \\ ${ }^{b}$ Institute of Informatics, Mathematics and E-Studies, Šiauliai University \\ P. Višinskio str. 19, LT-77156 Šiauliai, Lithuania \\ ${ }^{c}$ Research Institute, Šiauliai University \\ Vilniaus str. 88, LT-76285 Šiauliai, Lithuania \\ E-mail: kestutis.janulis@gmail.com \\ E-mail: d.jurgaitis@cr.su.lt \\ E-mail: antanas.laurincikas@mif.vu.lt \\ E-mail(corresp.): renata.macaitiene@mi.su.lt
}

\begin{abstract}
In [5], it was proved that a collection consisting from Dirichlet $L$-functions and periodic Hurwitz zeta-functions is universal in the sense that the shifts of those functions approximate simultaneously a given collection of analytic functions. In the paper, we prove theorems on the universality of composite functions of the above collection.
\end{abstract}

Keywords: Dirichlet $L$-function, Hurwitz zeta-function, mixed joint universality, periodic Hurwitz zeta-function, universality.

AMS Subject Classification: 11M06; 11M41.

\section{Introduction}

In [16], Voronin discovered the universality property of the Riemann zetafunction $\zeta(s), s=\sigma+i t$, on the approximation of analytic functions from a wide class by shifts $\zeta(s+i \tau), \tau \in \mathbb{R}$. At the moment, it is known that the majority of zeta and $L$-functions are universal in the above sense. Also, some zeta and $L$-functions are jointly universal: their shifts approximate simultaneously a given collection of analytic functions. A series of works are devoted to mixed joint universality when a collection of analytic functions are approximated simultaneously by shifts of zeta-functions with Euler product and without Euler product. The first result in this direction belongs to H. Mishou who proved [14] the joint universality of the function $\zeta(s)$ and the Hurwitz zeta-function $\zeta(s, \alpha)$ 
with transcendental parameter $\alpha$. This result has been generalized in [6] for a periodic zeta and a periodic Hurwitz zeta-functions. In [7], the mixed joint universality has been obtained for a wide collection consisting from periodic zeta and periodic Hurwitz zeta-functions. We remind that the periodic Hurwitz zeta-function $\zeta(s, \alpha ; \mathfrak{a})$, where $\alpha, 0<\alpha \leq 1$, is a fixed parameter and $\mathfrak{a}=\left\{a_{m}: m \in \mathbb{N}_{0}=\mathbb{N} \cup\{0\}\right\}$ is a periodic sequence of complex numbers, is a generalization of the classical Hurwitz zeta-function $\zeta(s, \alpha)$ when $a_{m} \equiv 1$, and is defined, for $\sigma>1$, by the series

$$
\zeta(s, \alpha ; \mathfrak{a})=\sum_{m=0}^{\infty} \frac{a_{m}}{(m+\alpha)^{s}},
$$

and by analytic continuation elsewhere. In [2], the mixed joint universality for a system of functions

$$
\zeta(s), \zeta\left(s, \alpha_{1}, \mathfrak{a}_{11}\right), \ldots, \zeta\left(s, \alpha_{1}, \mathfrak{a}_{1 l_{1}}\right), \ldots, \zeta\left(s, \alpha_{r}, \mathfrak{a}_{r 1}\right), \ldots, \zeta\left(s, \alpha_{r}, \mathfrak{a}_{r l_{r}}\right)
$$

has been considered. In a series of papers $[11,12,15]$, the Riemann zeta-function has been replaced by zeta-functions of certain cusp forms. In [5], in place of the function $\zeta(s)$ a collection of Dirichlet $L$-functions $L(s, \chi)$ has been put. We will state the latter result.

Let $D=\left\{s \in \mathbb{C}: \frac{1}{2}<\sigma<1\right\}$. Denote by $\mathcal{K}$ the class of compact subsets of the strip $D$ with connected complements, and by $H_{0}(K)$ and $H(K)$, $K \in \mathcal{K}$, the classes of continuous non-vanishing and continuous on $K$ functions, respectively, which are analytic in the interior of $K$. Let meas $A$ be the Lebesgue measure of a measurable set $A \subset \mathbb{R}$. Suppose that $\mathfrak{a}_{j l}=\left\{a_{m j l}: m \in \mathbb{N}_{0}\right\}$ is a periodic sequence of complex numbers with minimal period $k_{j l} \in \mathbb{N}, j=$ $1, \ldots, r, l=1, \ldots, l_{j}$. Let $k_{j}$ be the least common multiple of the periods $k_{j 1}, \ldots, k_{j l_{j}}$, and

$$
A_{j}=\left(\begin{array}{cccc}
a_{1 j 1} & a_{1 j 2} & \ldots & a_{1 j l_{j}} \\
a_{2 j 1} & a_{2 j 2} & \ldots & a_{2 j l_{j}} \\
\ldots & \ldots & \ldots & \ldots \\
a_{k_{j} j 1} & a_{k_{j} j 2} & \ldots & a_{k_{j} j l_{j}}
\end{array}\right), \quad j=1, \ldots, r .
$$

Then, in [5], the following theorem has been proved.

Theorem 1. Suppose that $\chi_{1}, \ldots, \chi_{d}$ are pairwise non-equivalent Dirichlet characters, the numbers $\alpha_{1}, \ldots, \alpha_{r}$ are algebraically independent over the field of rational numbers $\mathbb{Q}$, and that $\operatorname{rank}\left(A_{j}\right)=l_{j}, j=1, \ldots, r$. For $j=1, \ldots, d$, let $K_{j} \in \mathcal{K}$ and $f_{j} \in H_{0}\left(K_{j}\right)$, and, for $j=1, \ldots, r, l=1, \ldots, l_{j}$, let $K_{j l} \in \mathcal{K}$ and $f_{j l} \in H\left(K_{j l}\right)$. Then, for every $\varepsilon>0$,

$$
\begin{array}{r}
\liminf _{T \rightarrow \infty} \frac{1}{T} \text { meas }\left\{\tau \in[0, T]: \sup _{1 \leq j \leq d} \sup _{s \in K_{j}}\left|L\left(s+i \tau, \chi_{j}\right)-f_{j}(s)\right|<\varepsilon,\right. \\
\left.\sup _{1 \leq j \leq r} \sup _{1 \leq l \leq l_{j}} \sup _{s \in K_{j l}}\left|\zeta\left(s+i \tau, \alpha_{j} ; \mathfrak{a}_{j l}\right)-f_{j l}(s)\right|<\varepsilon\right\}>0 .
\end{array}
$$


Denote by $H(D)$ the space of analytic on $D$ functions equipped with the topology of uniform convergence on compacta. In [8] and [10], the Voronin theorem has been generalized for $F(\zeta(s))$ with certain operators $F: H(D) \rightarrow$ $H(D)$, in [9], the universality of $F(\zeta(s), \zeta(s, \alpha))$ has been studied with operators $F: H^{2}(D) \rightarrow H(D)$. The papers [3] and [4] are devoted to the universality of the functions $F\left(L\left(s, \chi_{1}\right), \ldots, L\left(s, \chi_{r_{1}}\right), \zeta\left(s, \alpha_{1}\right), \ldots, \zeta\left(s, \alpha_{r_{2}}\right)\right)$ for some operators $F: H^{r_{1}+r_{2}}(D) \rightarrow H(D)$. The aim of the present paper is the universality of composite functions of a collection of $L$ and zeta-functions in Theorem 1 , i.e., we consider the universality of the functions

$$
\begin{array}{r}
F\left(L\left(s, \chi_{1}\right), \ldots, L\left(s, \chi_{d}\right), \zeta\left(s, \alpha_{1} ; \mathfrak{a}_{11}\right), \ldots, \zeta\left(s, \alpha_{1} ; \mathfrak{a}_{1 l_{1}}\right), \ldots,\right. \\
\left.\zeta\left(s, \alpha_{r} ; \mathfrak{a}_{r 1}\right), \ldots, \zeta\left(s, \alpha_{r} ; \mathfrak{a}_{r l_{r}}\right)\right)
\end{array}
$$

for some operators $F$.

First we deal with approximation of functions from the class $H(K), K \in \mathcal{K}$. Let, for brevity, $v=d+l_{1}+\cdots+l_{r}$. We say that the operator $F: H^{v}(D) \rightarrow$ $H(D)$ belongs to the class $\operatorname{Lip}\left(\beta_{1}, \ldots, \beta_{v}\right), \beta_{1}>0, \ldots, \beta_{v}>0$, if the following hypotheses are satisfied:

$1^{\circ}$ For every polynomial $p=p(s)$ and all sets $K_{1}, \ldots, K_{d} \in \mathcal{K}$, there exists an element $g=\left(g_{1}, \ldots, g_{d}, g_{11}, \ldots, g_{1 l_{1}}, \ldots, g_{r 1}, \ldots, g_{r l_{r}}\right) \in F^{-1}\{p\} \subset H^{v}(D)$ such that $g_{j} \neq 0$ on $K_{j}, j=1, \ldots, d$;

$2^{\circ}$ For all $K \in \mathcal{K}$, there exist a constant $c>0$ and sets $K_{1}, \ldots, K_{v} \in \mathcal{K}$ such that, for all $\left(g_{j 1}, \ldots, g_{j v}\right) \in H^{v}(D), j=1,2$,

$$
\begin{array}{r}
\sup _{s \in K}\left|F\left(g_{11}(s), \ldots, g_{1 v}(s)\right)-F\left(g_{21}(s), \ldots, g_{2 v}(s)\right)\right| \\
\leq c \sup _{1 \leq j \leq v} \sup _{s \in K_{j}}\left|g_{1 j}(s)-g_{2 j}(s)\right|^{\beta_{j}} .
\end{array}
$$

Theorem 2. Suppose that $\chi_{1}, \ldots, \chi_{d}$ are pairwise non-equivalent Dirichlet characters, the numbers $\alpha_{1}, \ldots, \alpha_{r}$ are algebraically independent over the field of rational numbers $\mathbb{Q}, \operatorname{rank}\left(A_{j}\right)=l_{j}, j=1, \ldots, r$, and that $F \in \operatorname{Lip}\left(\beta_{1}, \ldots, \beta_{v}\right)$. Let $K \in \mathcal{K}$ and $f(s) \in H(K)$. Then, for every $\varepsilon>0$,

$$
\begin{array}{r}
\liminf _{T \rightarrow \infty} \frac{1}{T} \operatorname{meas}\left\{\tau \in[0, T]: \sup _{s \in K} \mid F\left(L\left(s+i \tau, \chi_{1}\right), \ldots, L\left(s+i \tau, \chi_{d}\right),\right.\right. \\
\zeta\left(s+i \tau, \alpha_{1} ; \mathfrak{a}_{11}\right), \ldots, \zeta\left(s+i \tau, \alpha_{1} ; \mathfrak{a}_{1 l_{1}}\right), \ldots, \zeta\left(s+i \tau, \alpha_{r} ; \mathfrak{a}_{r 1}\right), \ldots, \\
\left.\left.\zeta\left(s+i \tau, \alpha_{r} ; \mathfrak{a}_{r l_{r}}\right)\right)-f(s) \mid<\varepsilon\right\}>0 .
\end{array}
$$

We give an example of the operator $F \in \operatorname{Lip}\left(\beta_{1}, \ldots, \beta_{v}\right)$. Let, for $\left(g_{1}, \ldots, g_{d}\right.$, $\left.g_{11}, \ldots, g_{1 l_{1}}, \ldots, g_{r 1}, \ldots, g_{r l_{r}}\right) \in H^{v}(D)$,

$$
\begin{gathered}
F\left(g_{1}, \ldots, g_{d}, g_{11}, \ldots, g_{1 l_{1}}, \ldots, g_{r 1}, \ldots, g_{r l_{r}}\right)=c_{1} g_{1}^{\left(n_{1}\right)}+\cdots+c_{d} g_{d}^{\left(n_{d}\right)} \\
\quad+c_{11} g_{11}^{\left(n_{11}\right)}+\cdots+c_{1 l_{1}} g_{1 l_{1}}^{\left(n_{1 l_{1}}\right)}+\cdots+c_{r 1} g_{r 1}^{\left(n_{r 1}\right)}+\cdots+c_{r l_{r}} g_{r l_{r}}^{\left(n_{r l_{r}}\right)}
\end{gathered}
$$

where $c_{1}, \ldots, c_{d}, c_{11}, \ldots, c_{1 l_{1}}, \ldots, c_{r 1}, \ldots, c_{r l_{r}} \in \mathbb{C} \backslash\{0\}, n_{1}, \ldots, n_{d}, n_{11}, \ldots$, $n_{1 l_{1}}, \ldots, n_{r 1}, \ldots, n_{r l_{r}} \in \mathbb{N}$, and $f^{(n)}$ denotes the $n$th derivative of $f$. It is not 
difficult to see that, for each polynomial $p=p(s)$ and all sets $K_{1}, \ldots, K_{d} \in \mathcal{K}$, there exists an element $\underline{g} \in F^{-1}\{p\}$ such that $g_{j}(s) \neq 0$ on $K_{j}, j=1, \ldots, d$. For example, if

$$
p(s)=a_{k} s^{k}+a_{k-1} s^{k-1}+\cdots+a_{0}, \quad a_{k} \neq 0,
$$

we can take $\underline{g}=\left(1, \ldots, 1,1, \ldots, 1,1 \ldots, g_{r l_{r}}\right)$, where

$$
g_{r l_{r}}(s)=\frac{1}{c_{r l_{r}}}\left(\frac{a_{n} s^{k+n_{r l_{r}}}}{(k+1) \cdots\left(k+n_{r l_{r}}\right)}+\cdots+\frac{a_{0} s^{n_{r l_{r}}}}{1 \cdots n_{r l_{r}}}\right) .
$$

Thus, hypothesis $1^{\circ}$ of the class $\operatorname{Lip}\left(\beta_{1}, \ldots, \beta_{v}\right)$ is satisfied.

Hypothesis $2^{\circ}$ of the class $\operatorname{Lip}\left(\beta_{1}, \ldots, \beta_{v}\right)$ follows from the Cauchy integral formula. We write $F$ in a more convenient form

$$
F\left(g_{1}, \ldots, g_{v}\right)=\sum_{j=1}^{v} c_{j} g_{j}^{\left(n_{j}\right)}
$$

Let $K \in \mathcal{K}$, and $K \subset G \subset K_{1}$, where $G$ is an open set and $K_{1} \in \mathcal{K}$. Moreover, let $L$ be a simple closed contour lying in $K_{1} \backslash G$ and containing inside the $K$. Then the Cauchy integral formula shows that, for $\left(g_{j 1}, \ldots, g_{j v}\right) \in H^{v}(D)$, $j=1,2$, and $s \in K$,

$$
\begin{array}{r}
\left|F\left(g_{11}(s), \ldots, g_{1 v}(s)\right)-F\left(g_{21}(s), \ldots, g_{2 v}(s)\right)\right|=\left|\sum_{j=1}^{v} c_{j} \frac{n_{j} !}{2 \pi i} \int_{L} \frac{g_{1 j}(z)-g_{2 j}(z)}{(z-s)^{n_{j}+1}} \mathrm{~d} z\right| \\
\leq \sum_{j=1}^{v}\left|c_{j}\right| C_{j} \sup _{s \in L}\left|g_{1 j}(s)-g_{2 j}(s)\right| \leq c \sup _{1 \leq j \leq v} \sup _{s \in K_{1}}\left|g_{1 j}(s)-g_{2 j}(s)\right|
\end{array}
$$

with some constants $C_{j}>0, j=1, \ldots, v$, and $c>0$. Thus we have that $F \in \operatorname{Lip}(1, \ldots, 1)$, and in this case, $K_{1}=\cdots=K_{v}=K_{1}$.

Now we give some other classes of operators $F$. Let

$$
S=\{g \in H(D): g(s) \neq 0 \text { or } g(s) \equiv 0\} .
$$

Moreover, $v_{1}=\sum_{j=1}^{r} l_{j}$.

Theorem 3. Suppose that the characters $\chi_{1}, \ldots, \chi_{d}$, the numbers $\alpha_{1}, \ldots, \alpha_{r}$ and the sequences $\mathfrak{a}_{j l}, j=1, \ldots, r, l=1, \ldots, l_{j}$, satisfy the hypotheses of Theorem 2, and that $F: H^{v}(D) \rightarrow H(D)$ be a continuous operator such that, for every open set $G \subset H(D)$, the set $\left(F^{-1} G\right) \cap\left(S^{d} \times H^{v_{1}}(D)\right)$ is not empty. Let $K \in \mathcal{K}$ and $f(s) \in H(K)$. Then the assertion of Theorem 2 is true.

We note that the hypothesis $\left(F^{-1} G\right) \cap\left(S^{d} \times H^{v_{1}}(D)\right) \neq \varnothing$ for every open set $G \subset H(D)$ is general but sufficiently complicated. Obviously, it is satisfied if every $g \in H(D)$ has a preimage in the set $S^{d} \times H^{v_{1}}(D)$. On the other hand, Theorem 3 implies the following modification of Theorem 2.

Theorem 4. Suppose that the characters $\chi_{1}, \ldots, \chi_{d}$, the numbers $\alpha_{1}, \ldots, \alpha_{r}$ and the sequences $\mathfrak{a}_{j l}, j=1, \ldots, r, l=1, \ldots, l_{j}$, satisfy the hypotheses of Theorem 2, and that $F: H^{v}(D) \rightarrow H(D)$ is a continuous operator such that, for every polynomial $p=p(s)$, the set $\left(F^{-1}\{p\}\right) \cap\left(S^{d} \times H^{v_{1}}(D)\right)$ is not empty. Let $K \in \mathcal{K}$ and $f(s) \in H(K)$. Then the assertion of Theorem 2 is true. 
Clearly, hypothesis $2^{\circ}$ of the class $\operatorname{Lip}\left(\beta_{1}, \ldots, \beta_{v}\right)$ implies the continuity of $F$. However, hypothesis $1^{\circ}$ is weaker than the requirement $\left(F^{-1}\{p\}\right) \cap\left(S^{d} \times\right.$ $\left.H^{v_{1}}(D)\right) \neq \varnothing$.

Non-vanishing of the polynomial $p(s)$ in a bounded region can be controlled by its constant term. Therefore, sometimes it is more convenient to consider operators $F$ on the space $H^{v}\left(D_{V}, D\right)=H^{d}\left(D_{V}\right) \times H^{v_{1}}(D)$, where, for $V>0$, $D_{V}=\left\{s \in \mathbb{C}: \frac{1}{2}<\sigma<1,|t|<V\right\}$. Analogically, let

$$
S_{V}=\left\{g \in H\left(D_{V}\right): g(s) \neq 0 \text { or } g(s) \equiv 0\right\} .
$$

Then we have the following result.

Theorem 5. Suppose that the characters $\chi_{1}, \ldots, \chi_{d}$, the numbers $\alpha_{1}, \ldots, \alpha_{r}$ and the sequences $\mathfrak{a}_{j l}, j=1, \ldots, r, l=1, \ldots, l_{j}$, satisfy the hypotheses of Theorem 2, $K \in \mathcal{K}, f(s) \in H(K)$ and $V>0$ is such that $K \subset D_{V}$. Let $F: H^{v}\left(D_{V}, D\right) \rightarrow H\left(D_{V}\right)$ be a continuous operator such that, for every polynomial $p=p(s)$, the set $\left(F^{-1}\{p\}\right) \cap\left(S_{V}^{d} \times H^{v_{1}}(D)\right)$ is not empty. Then the assertion of Theorem 2 is true.

For example, Theorem 5 can be applied for the operator

$$
F\left(g_{1}, \ldots, g_{v}\right)=c_{1} g_{1}^{\left(n_{1}\right)}+\cdots+c_{d} g_{d}^{\left(n_{d}\right)}, \quad n_{1}, \ldots, n_{d} \in \mathbb{N} .
$$

Now we consider approximation of analytic functions from the image of the set $S^{d} \times H^{v_{1}}(S)$ of the operator $F: H^{v}(D) \rightarrow H(D)$.

Theorem 6. Suppose that the characters $\chi_{1}, \ldots, \chi_{d}$, the numbers $\alpha_{1}, \ldots, \alpha_{r}$ and the sequences $\mathfrak{a}_{j l}, j=1, \ldots, r, l=1, \ldots, l_{j}$, satisfy the hypotheses of Theorem 2, and that $F: H^{v}(D) \rightarrow H(D)$ is a continuous operator. Let $K \subset D$ be a compact subset, and $f(s) \in F\left(S^{d} \times H^{v_{1}}(D)\right)$. Then the assertion of Theorem 2 is true.

It is not easy to describe the set $F\left(S^{d} \times H^{v_{1}}(D)\right)$. The next theorem is an example with sufficiently simple set contained in $F\left(S^{d} \times H^{v_{1}}(D)\right)$.

Suppose that $a_{1}, \ldots, a_{k} \in \mathbb{C}$ are pairwise different numbers, and

$$
H_{k}(D)=\left\{g \in H(D):\left(g(s)-a_{j}\right)^{-1} \in H(D), j=1, \ldots, k\right\} .
$$

Theorem 7. Suppose that the characters $\chi_{1}, \ldots, \chi_{d}$, the numbers $\alpha_{1}, \ldots, \alpha_{r}$ and the sequences $\mathfrak{a}_{j l}, j=1, \ldots, r, l=1, \ldots, l_{j}$, satisfy the hypotheses of Theorem 2, and that $F: H^{v}(D) \rightarrow H(D)$ is a continuous operator such that $F\left(S^{d} \times H^{v_{1}}(D)\right) \supset H_{k}(D)$. For $k=1$, let $K \in \mathcal{K}, f(s) \in H(K)$ and $f(s) \neq a_{1}$ on $K$. For $k \geq 2$, let $K \subset D$ be an arbitrary compact subset, and $f(s) \in H_{k}(D)$. Then the assertion of Theorem 2 is true.

For example, let $k=2$ and $a_{1}=1, a_{2}=-1$. Then Theorem 7 implies the universality of the function

$$
\begin{aligned}
\sin \left(L\left(s, \chi_{1}\right)+\cdots+L\left(s, \chi_{d}\right)+\zeta\right. & \left(s, \alpha_{1} ; \mathfrak{a}_{11}\right)+\cdots+\zeta\left(s, \alpha_{1} ; \mathfrak{a}_{1 l_{1}}\right)+\cdots \\
& \left.+\zeta\left(s, \alpha_{r} ; \mathfrak{a}_{r 1}\right)+\cdots+\zeta\left(s, \alpha_{r} ; \mathfrak{a}_{r l_{r}}\right)\right) .
\end{aligned}
$$


For this, it suffices to consider the equation

$$
\frac{e^{i \Sigma(s)}-e^{-i \Sigma(s)}}{2 i}=f, \quad f \in H(D), a_{1}=1, a_{2}=-1,
$$

where $\Sigma(s)$ is the sum under the sign of $\sin$.

\section{Proof of Theorem 2}

Theorem 2 is a result of Theorem 1 , properties of the class $\operatorname{Lip}\left(\beta_{1}, \ldots, \beta_{v}\right)$ and of the Mergelyan theorem on the approximation of analytic functions by polynomials. We state this theorem in the form of the next lemma.

Lemma 1. Suppose that $K \subset \mathbb{C}$ is a compact subset with connected complement, and $f(s)$ is a continuous function on $K$ which is analytic in the interior of $K$. Then, for every $\varepsilon>0$, there exists a polynomial $p(s)$ such that

$$
\sup _{s \in K}|f(s)-p(s)|<\varepsilon .
$$

Proof of the lemma can be found in [13] and [17].

Proof of Theorem 2. Lemma 1 implies the existence of the polynomial $p=p(s)$ such that

$$
\sup _{s \in K}|f(s)-p(s)|<\frac{\varepsilon}{2} .
$$

Using hypothesis $1^{\circ}$ of the class $\operatorname{Lip}\left(\beta_{1}, \ldots, \beta_{v}\right)$, we have that, for all sets $K_{1}, \ldots, K_{d} \in \mathcal{K}$, there exists an element $\left(g_{1}, \ldots, g_{d}, g_{11}, \ldots, g_{1 l_{1}}, \ldots, g_{r 1}, \ldots\right.$, $\left.g_{r l_{r}}\right) \in F^{-1}\{p\}$ such that $g_{j}(s) \neq 0$ on $K_{j}, j=1, \ldots, d$. Suppose that $\tau \in \mathbb{R}$ satisfies the inequalities

$$
\begin{aligned}
& \sup _{1 \leq j \leq d} \sup _{s \in K_{j}}\left|L\left(s+i \tau, \chi_{j}\right)-f_{j}(s)\right|<c^{-\frac{1}{\beta}}\left(\frac{\varepsilon}{4}\right)^{\frac{1}{\beta}}, \\
& \sup _{1 \leq j \leq r} \sup _{1 \leq l \leq l_{j}} \sup _{s \in K_{j l}}|| \zeta\left(s+i \tau, \alpha_{j} ; \mathfrak{a}_{j l}\right)-f_{j l}(s) \mid<c^{-\frac{1}{\beta}}\left(\frac{\varepsilon}{4}\right)^{\frac{1}{\beta}},
\end{aligned}
$$

where the sets $K_{1}, \ldots, K_{d}, K_{11}, \ldots, K_{1 l_{1}}, \ldots, K_{r 1}, \ldots, K_{r l_{r}} \in \mathcal{K}$ correspond the set $K$ in hypothesis $2^{\circ}$ of the class $\operatorname{Lip}\left(\beta_{1}, \ldots, \beta_{v}\right)$, and $\beta=\min _{1 \leq j \leq v} \beta_{j}$, with notation $K_{1 l}=K_{d+l}, j=1, \ldots, l_{1}, \ldots, K_{r l}=K_{d+l_{1}+\cdots+l_{r-1}+l}, l=$ $1, \ldots, l_{r}$. Then, in view of Theorem 1 , the set of $\tau$ satisfying inequalities $(2.2)$ and $(2.3)$ has a positive lower density. Moreover, hypothesis $2^{\circ}$ of the class $\operatorname{Lip}\left(\beta_{1}, \ldots, \beta_{v}\right)$ shows that, for such $\tau$,

$$
\begin{aligned}
& \sup _{s \in K}\left|F(\underline{L}(s+i \tau, \underline{\chi}, \underline{\alpha}, \underline{\mathfrak{a}}))-p(s) \leq \sup _{1 \leq j \leq d} \sup _{s \in K_{j}}\right| L\left(s+i \tau, \chi_{j}\right)-\left.f_{j}(s)\right|^{\beta_{j}} \\
& +c \sup _{1 \leq j \leq r} \sup _{1 \leq l \leq l_{j}} \sup _{s \in K_{j l}}\left|\zeta\left(s+i \tau, \alpha_{j} ; \mathfrak{a}_{j l}\right)-f_{j l}(s)\right|^{\beta_{j l}} \leq 2 c c^{-\frac{\beta}{\beta}}\left(\frac{\varepsilon}{4}\right)^{\frac{\beta}{\beta}}=\frac{\varepsilon}{2} .
\end{aligned}
$$


Here $\underline{\chi}=\left(\chi_{1}, \ldots, \chi_{d}\right), \underline{\alpha}=\left(\alpha_{1}, \ldots, \alpha_{r}\right), \underline{\mathfrak{a}}=\left(\mathfrak{a}_{11}, \ldots, \mathfrak{a}_{1, l_{1}}, \ldots, \mathfrak{a}_{r 1}, \ldots\right.$, $\left.\mathfrak{a}_{r l_{r}}\right)$ and

$$
\begin{array}{r}
\underline{L}(s+i \tau, \underline{\chi}, \underline{\alpha}, \underline{\mathfrak{a}})=\left(L\left(s, \chi_{1}\right), \ldots, L\left(s, \chi_{d}\right), \zeta\left(s, \alpha_{1} ; \mathfrak{a}_{11}\right), \ldots, \zeta\left(s, \alpha_{1} ; \mathfrak{a}_{1 l_{1}}\right), \ldots,\right. \\
\left.\zeta\left(s, \alpha_{r} ; \mathfrak{a}_{r 1}\right), \ldots, \zeta\left(s, \alpha_{r} ; \mathfrak{a}_{r l_{r}}\right)\right),
\end{array}
$$

and $\beta_{1 l}=\beta_{d+l}, l=1, \ldots, l_{1}, \ldots, \beta_{r l}=\beta_{d+l_{1}+\cdots+l_{r-1}+l}, l=1, \ldots, l_{r}$. Thus, by the above remark,

$$
\liminf _{T \rightarrow \infty} \frac{1}{T} \operatorname{meas}\left\{\tau \in[0, T]: \sup _{s \in K}|F(\underline{L}(s+i \tau, \underline{\chi}, \underline{\alpha}, \underline{\mathfrak{a}}))-p(s)|<\frac{\varepsilon}{2}\right\}>0 .
$$

Combining this with inequality (2.1) proves the theorem.

\section{Elements of probability theory}

For the proof of Theorems $3-7$, we apply a probabilistic approach based on limit theorems for weakly convergent probability measures in the space of analytic functions. We start with a limit theorem for $\underline{L}(s+i \tau, \underline{\chi}, \underline{\alpha}, \underline{\mathfrak{a}})$ obtained in [5], Theorem 2.

Denote by $\mathcal{B}(X)$ the Borel $\sigma$-field of the space $X$. Let $\gamma=\{s \in \mathbb{C}:|s|=1\}$ be the unit circle on the complex plane, and

$$
\Omega=\prod_{p \in \mathcal{P}} \gamma_{p}, \quad \hat{\Omega}=\prod_{m \in \mathbb{N}_{0}} \gamma_{m},
$$

where $\mathcal{P}$ is the set of all prime numbers, and $\gamma_{p}=\gamma$ for all $p \in \mathcal{P}$ and $\gamma_{m}=\gamma$ for all $m \in \mathbb{N}_{0}$. In view of the classical Tikhonov theorem, the tori $\Omega$ and $\hat{\Omega}$ with the product topology and pointwise multiplication are compact topological Abelian groups. Moreover, let

$$
\underline{\Omega}=\Omega \times \hat{\Omega}_{1} \times \cdots \times \hat{\Omega}_{r}
$$

where $\hat{\Omega}_{j}=\hat{\Omega}$ for all $j=1, \ldots, r$. Then again $\underline{\Omega}$ is a compact topological Abelian group. This leads to the probability space $\left(\underline{\Omega}, \mathcal{B}(\underline{\Omega}), \underline{m}_{H}\right)$, where $\underline{m}_{H}$ is the probability Haar measure on $(\underline{\Omega}, \mathcal{B}(\underline{\Omega}))$. Denote by $\omega(p)$ the projection of the element $\omega \in \Omega$ to the coordinate space $\gamma_{p}, p \in \mathcal{P}$, and by $\hat{\omega}_{j}(m)$ the projection of an element $\hat{\omega}_{j} \in \hat{\Omega}_{j}$ to the coordinate space $\gamma_{m}, m \in \mathbb{N}_{0}, j=$ $1, \ldots, r$. Let $p^{k} \| m$ mean that $p^{k} \mid m$ but $p^{k+1} \nmid m$. Extend the function $\omega(p)$ to the set $\mathbb{N}$ by taking

$$
\omega(m)=\prod_{p^{k} \| m} \omega^{k}(m), \quad m \in \mathbb{N} .
$$

Denote by $\underline{\omega}=\left(\omega, \hat{\omega}_{1}, \ldots, \hat{\omega}_{r}\right)$ the elements of $\underline{\Omega}$, and, on the probability space $\left(\underline{\Omega}, \mathcal{B}(\underline{\Omega}), \underline{m}_{H}\right)$, define the $H^{v}(D)$-valued random element $\underline{L}(s, \underline{\chi}, \underline{\alpha}, \underline{\omega}, \underline{\mathfrak{a}})$ by the formula

$$
\begin{array}{r}
\underline{L}(s, \underline{\chi}, \underline{\alpha}, \underline{\omega}, \underline{\mathfrak{a}})=\left(L\left(s, \omega, \chi_{1}\right), \ldots, L\left(s, \omega, \chi_{d}\right), \zeta\left(s, \alpha_{1}, \omega_{1} ; \mathfrak{a}_{11}\right), \ldots,\right. \\
\left.\zeta\left(s, \alpha_{1}, \omega_{1} ; \mathfrak{a}_{1 l_{1}}\right), \ldots, \zeta\left(s, \alpha_{r}, \omega_{r} ; \mathfrak{a}_{r 1}\right), \ldots, \zeta\left(s, \alpha_{r}, \omega_{r} ; \mathfrak{a}_{r l_{r}}\right)\right),
\end{array}
$$


where

$$
L\left(s, \omega, \chi_{j}\right)=\sum_{m=1}^{\infty} \frac{\chi_{j}(m) \omega(m)}{m^{s}}, \quad j=1, \ldots, d,
$$

and

$$
\zeta\left(s, \alpha_{j}, \omega_{j} ; \mathfrak{a}_{j l}\right)=\sum_{m=1}^{\infty} \frac{a_{m j l} \omega_{j}(m)}{\left(m+\alpha_{j}\right)^{s}}, \quad j=1, \ldots, r, l=1, \ldots, l_{j} .
$$

We note that the latter series are uniformly convergent on compact subsets of $D$ for almost all $\underline{\omega} \in \underline{\Omega}$. Moreover, for almost $\underline{\omega} \in \underline{\Omega}, L\left(s, \omega, \chi_{j}\right)$ can be written in the form

$$
L\left(s, \omega, \chi_{j}\right)=\prod_{p}\left(1-\frac{\chi_{j}(p) \omega(p)}{p^{s}}\right)^{-1} .
$$

Denote by $P_{\underline{L}}$ the distribution of the random element $\underline{L}(s, \underline{\chi}, \underline{\alpha}, \underline{\omega}, \underline{\mathfrak{a}})$, i.e., the probability measure

$$
P_{\underline{L}}(A)=\underline{m}_{H}(\underline{\omega} \in \underline{\Omega}: \underline{L}(s, \underline{\chi}, \underline{\alpha}, \underline{\omega}, \underline{\mathfrak{a}}) \in A), \quad A \in \mathcal{B}\left(H^{v}(D)\right) .
$$

Then we have the following limit theorem [5].

Lemma 2. Suppose that the numbers $\alpha_{1}, \ldots, \alpha_{r}$ are algebraically independent over $\mathbb{Q}$. Then

$$
P_{T}(A) \stackrel{\text { def }}{=} \frac{1}{T} \operatorname{meas}\{\tau \in[0, T]: \underline{L}(s+i \tau, \underline{\chi}, \underline{\alpha}, \underline{\mathfrak{a}}) \in A\}, \quad A \in \mathcal{B}\left(H^{v}(D)\right)
$$

converges weakly to $P_{\underline{L}}$ as $T \rightarrow \infty$.

For the proof a limit theorem for composite function $F(\underline{L}(s, \underline{\chi}, \underline{\alpha}, \underline{\mathfrak{a}}))$, we will apply an assertion on the preservation of the weak convergence under mappings. Let $X_{1}$ and $X_{2}$ be two metric spaces, and let $u: X_{1} \rightarrow X_{2}$ be a $\left(\mathcal{B}\left(X_{1}\right), \mathcal{B}\left(X_{2}\right)\right)$-measurable mapping, i.e.,

$$
u^{-1} \mathcal{B}\left(X_{2}\right) \subset \mathcal{B}\left(X_{1}\right)
$$

Then every probability measure $P$ on $\left(X_{1}, \mathcal{B}\left(X_{1}\right)\right)$ induces the unique probability measure $P u^{-1}$ on $\left(X_{2}, \mathcal{B}\left(X_{2}\right)\right)$ defined by

$$
P u^{-1}(A)=P\left(u^{-1} A\right), \quad A \in \mathcal{B}\left(X_{2}\right) .
$$

It is well known that the continuity of $u$ implies its $\left(\mathcal{B}\left(X_{1}\right), \mathcal{B}\left(X_{2}\right)\right)$-measurability.

Lemma 3. Suppose that $P_{n}$ converges weakly to $P$ as $n \rightarrow \infty$, and that the mapping $u: X_{1} \rightarrow X_{2}$ is continuous. Then $P_{n} u^{-1}$ converges weakly to $P u^{-1}$ as $n \rightarrow \infty$.

Proof of the lemma is given in [1]. 
Lemma 4. Suppose that the numbers $\alpha_{1}, \ldots, \alpha_{r}$ are algebraically independent over $\mathbb{Q}$ and that the operator $F: H^{v}(D) \rightarrow H(D)$ is continuous. Then

$$
P_{T, F}(A) \stackrel{\text { def }}{=} \frac{1}{T} \text { meas }\{\tau \in[0, T]: F(\underline{L}(s+i \tau, \underline{\chi}, \underline{\alpha}, \underline{\mathfrak{a}})) \in A\}, \quad A \in \mathcal{B}\left(H^{v}(D)\right),
$$

converges weakly to $P_{\underline{L}} F^{-1}$ as $T \rightarrow \infty$.

Proof. The lemma is an immediate consequence of Lemmas 2 and 3.

For the proof of universality theorems for $F(\underline{L}(s+i \tau, \underline{\chi}, \underline{\alpha}, \underline{\mathfrak{a}}))$, we also need the explicit form of the support of the measure $P_{\underline{L}} F^{-1}$. We apply a result of [5] on the support of the measure $P_{\underline{L}}$.

Lemma 5. Suppose that $\chi_{1}, \ldots, \chi_{d}$ are pairwise non-equivalent Dirichlet characters, the numbers $\alpha_{1}, \ldots, \alpha_{r}$ are algebraically independent over $\mathbb{Q}$, and that $\operatorname{rank}\left(A_{j}\right)=l_{j}, j=1, \ldots, r$. Then the support of $P_{\underline{L}}$ is the set $S^{d} \times H^{v_{1}}(D)$.

Lemma 6. Suppose that the hypotheses of Lemma 5 are satisfied, and that the operator $F: H^{v}(D) \rightarrow H(D)$ is continuous. Then the support of $P_{\underline{L}} F^{-1}$ is the closure of the set $F\left(S^{d} \times H^{v_{1}}(D)\right)$.

Proof. Let $g$ be an arbitrary element of the set $F\left(S^{d} \times H^{v_{1}}(D)\right)$, and $G$ be any open neighbourhood of $g$. Then $F^{-1} G$ is an open neighbourhood of a certain element of the set $S^{d} \times H^{v_{1}}(D)$. Therefore, Lemma 5 and properties of a support imply that $P_{\underline{L}}\left(F^{-1} G\right)>0$, hence, $P_{\underline{L}} F^{-1}(G)>0$. Moreover, in virtue of Lemma 5 again,

$$
P_{\underline{L}} F^{-1}\left(F\left(S^{d} \times H^{v_{1}}(D)\right)\right)=P_{\underline{L}}\left(S^{d} \times H^{v_{1}}(D)\right)=1 .
$$

Since the support of $P_{\underline{L}} F^{-1}$ is a closed set, from this the lemma follows.

We also need one equivalent of the weak convergence of probability measures.

Lemma 7. Let $P_{n}, n \in \mathbb{N}$, and $P$ be probability measures on $(X, \mathcal{B}(X))$. Then, $P_{n}$, as $n \rightarrow \infty$, converges weakly to $P$ if and only if, for every open set $G \subset X$,

$$
\liminf _{n \rightarrow \infty} P_{n}(G) \geq P(G) .
$$

The lemma is a part of Theorem 2.1 from [1].

\section{Proofs of other universality theorems}

Proof of Theorem 3. It is not difficult to see that, under hypotheses of Theorem 3, the support of the measure $P_{\underline{L}} F^{-1}$ is the whole of $H(D)$. Indeed, if $\left(F^{-1} G\right) \cap\left(S^{d} \times H^{v_{1}}(D)\right) \neq \varnothing$ for every open set $G \subset H(D)$, then we have that, for every element $g \in H(D)$ and its open neighbourhood $G$, there exists an element of the set $F\left(S^{d} \times H^{v_{1}}(D)\right)$ which belongs to the set $G$. This shows 
that the set $F\left(S^{d} \times H^{v_{1}}(D)\right)$ is dense in $H(D)$. Since, by Lemma 6 , the support of $P_{\underline{L}} F^{-1}$ is the closure of $F\left(S^{d} \times H^{v_{1}}(D)\right)$, from this we obtain that the support of $P_{\underline{L}} F^{-1}$ is the whole of $H(D)$.

In view of Lemma 1 , there exists a polynomial $p(s)$ satisfying inequality (2.1). Define the set

$$
G=\left\{g \in H(D): \sup _{s \in K}|g(s)-p(s)|<\frac{\varepsilon}{2}\right\} .
$$

Obviously, $G$ is an open neighbourhood of $p(s)$ which is an element of the support of $P_{\underline{L}} F^{-1}$. Therefore, $P_{\underline{L}} F^{-1}(G)>0$, and Lemmas 4 and 7 imply the inequality

$$
\liminf _{T \rightarrow \infty} \frac{1}{T} \operatorname{meas}\{\tau \in[0, T]: F(\underline{L}(s+i \tau, \underline{\chi}, \underline{\alpha}, \underline{\mathfrak{a}})) \in G\}>0,
$$

or, by definition of $G$,

$$
\liminf _{T \rightarrow \infty} \frac{1}{T} \operatorname{meas}\left\{\tau \in[0, T]: \sup _{s \in K}|F(\underline{L}(s+i \tau, \underline{\chi}, \underline{\alpha}, \underline{\mathfrak{a}}))-p(s)|<\frac{\varepsilon}{2}\right\}>0 .
$$

Combining this with (2.1) gives the assertion of the theorem.

Proof of Theorem 4. Let $\left\{K_{l}: l \in \mathbb{N}\right\} \subset D$ be the sequence of compact subsets such that $K_{l} \subset K_{l+1}$ for all $l \in \mathbb{N}$,

$$
D=\bigcup_{l=1}^{\infty} K_{l}
$$

and, for every compact subset $K \subset D$, there exists $K_{l}$ such that $K \subset K_{l}$. For $g_{1}, g_{2} \in H(D)$, define

$$
\rho\left(g_{1}, g_{2}\right)=\sum_{l=1}^{\infty} 2^{-l} \frac{\sup _{s \in K_{l}}\left|g_{1}(s)-g_{2}(s)\right|}{1+\sup _{s \in K_{l}}\left|g_{1}(s)-g_{2}(s)\right|} .
$$

Then $\rho$ is a metric on $H(D)$ which induces its topology of uniform convergence on compacta. Moreover, from the definition of $\rho$ we have that the function $g_{2}$ approximates $g_{1}$ with suitable accuracy if $g_{2}$ approximate $g_{1}$ uniformly on $K_{l}$ for sufficiently large $l$. Obviously, we may choose the sets $K_{l}$ with connected complements, for example, we can take the closed rectangles. Thus, in $H(D)$, we can limit ourselves by approximation of functions on compact subsets with connected complements.

We will show that the hypotheses of the theorem imply those of Theorem 3. Let $G \subset H(D)$ be an arbitrary non-empty open set. Then, in view of Lemma 1 and the above remark on approximation in $H(D)$, there exists a polynomial $p(s) \in G$. Therefore, the hypothesis $\left(F^{-1}\{p\}\right) \cap\left(S^{d} \times H^{v_{1}}(D)\right) \neq \varnothing$ implies that of Theorem 3 that the set $\left(F^{-1} G\right) \cap\left(S^{d} \times H^{v_{1}}(D)\right)$ is non-empty. Thus, Theorem 4 is a corollary of Theorem 3 .

Proof of Theorem 5. We apply the arguments used in the proof of Theorem 3 with obvious modifications. 
Proof of Theorem 6. Since $f(s) \in F\left(S^{d} \times H^{v_{1}}(D)\right)$, it follows from Lemma 6 that $f(s)$ is an element of the support of the measure $P_{\underline{L}} F^{-1}$. Hence, $P_{\underline{L}} F^{-1}(G)$ $>0$ for

$$
G=\left\{g \in H(D): \sup _{s \in K}|g(s)-p(s)|<\varepsilon\right\} .
$$

Therefore, the theorem is a consequence of Lemmas 4 and 7.

Proof of Theorem \%. First suppose that $k=1$. By Lemma 1, there exists a polynomial $p(s)$ such that

$$
\sup _{s \in K}|f(s)-p(s)|<\frac{\varepsilon}{4}
$$

Since $f(s) \neq a_{1}$ on $K$, then also $p(s) \neq a_{1}$ on $K$ if $\varepsilon>0$ is rather small. Therefore, on $K$ we can define a continuous branch of the $\operatorname{logarithm} \log (p(s)-$ $a_{1}$ ) which will be an analytic function in the interior of $K$. Again by Lemma 1 , we can find a polynomial $q(s)$ such that

$$
\sup _{s \in K}\left|p(s)-a_{1}-e^{q(s)}\right|<\frac{\varepsilon}{4}
$$

Let, for brevity, $g_{a_{1}}(s)=a_{1}+e^{q(s)}$. Then, clearly, $g_{a_{1}}(s) \in H(D)$, and $g_{a_{1}}(s) \neq$ $a_{1}$ on $D$. Thus, $g_{a_{1}}(s) \in H_{1}(D)$. In view of Lemma 6 , the support of the measure $P_{L} F^{-1}$ contains the closure of the set $H_{1}(D)$. Therefore, the function $g_{a_{1}}(s)$ is an element of the support of the measure $P_{\underline{L}} F^{-1}$. Hence, $P_{\underline{L}} F^{-1}(G)>$ 0 , where

$$
G=\left\{g \in H(D): \sup _{s \in K}\left|g(s)-g_{a_{1}}(s)\right|<\frac{\varepsilon}{2}\right\} .
$$

This together with Lemmas 4 and 7 shows that

$$
\liminf _{T \rightarrow \infty} \frac{1}{T} \text { meas }\left\{\tau \in[0, T]: \sup _{s \in K}\left|F(\underline{L}(s+i \tau, \underline{\chi}, \underline{\alpha}, \underline{\mathfrak{a}}))-g_{a_{1}}(s)\right|<\frac{\varepsilon}{2}\right\}>0 .
$$

Inequalities (4.1) and (4.2) imply that

$$
\sup _{s \in K}\left|f(s)-g_{a_{1}}(s)\right|<\frac{\varepsilon}{2} .
$$

This and (4.3) yield the assertion of the theorem in the case $k=1$.

The case $k \geq 2$ is contained in Theorem 6 .

\section{References}

[1] P. Billingsley. Convergence of Probability Measures. Wiley, New York, 1968.

[2] J. Genys, R. Macaitienè, S. Račkauskienè and D. Šiaučiūnas. A mixed joint universality theorem for zeta-functions. Math. Modelling and Analysis, 15(4):431446, 2010.

[3] K. Janulis. Remarks on the joint universality of Dirichlet $L$-functions and Hurwitz zeta-functions. Šiauliai Math. Semin., 9(17):61-70, 2014. 
[4] K. Janulis and A. Laurinčikas. Joint universality of Dirichlet $L$-functions and Hurwitz zeta-functions. Ann. Univ. Sci. Budapest, Sect. Comp., 39:203-214, 2013.

[5] K. Janulis, A. Laurinčikas, R. Macaitienè and D. Šiaučiūnas. Joint universality of Dirichlet $L$-functions and periodic Hurwitz zeta-functions. Math. Modelling and Analysis, 17(5):673-685, 2012.

[6] R. Kačinskaitè and A. Laurinčikas. The joint distribution of periodic zetafunctions. Studia Scientiarum Mathematicarum Hungarica, 48(2):257-279, 2011. http://dx.doi.org/10.1556/SScMath.48.2011.2.1162.

[7] A. Laurinčikas. Joint universality of zeta-functions with periodic coefficients. Izvestiya: Mathematics, $\mathbf{7 4}(3): 515-539,2010$. http://dx.doi.org/10.1070/IM2010v074n03ABEH002497.

[8] A. Laurinčikas. Universality of the Riemann zeta-function. Journal of Number Theory, 130(10):2323-2331, 2010. http://dx.doi.org/10.1016/j.jnt.2010.04.007.

[9] A. Laurinčikas. On joint universality of the Riemann zeta-function and Hurwitz zeta-functions. Journal of Number Theory, 132(12):2842-2853, 2012. http://dx.doi.org/10.1016/j.jnt.2012.05.026.

[10] A. Laurinčikas. Universality of composite functions. In K. Matsumoto et al.(Ed.), Functions in Number Theory and Their Probabilistic Aspects, volume B34, pp. 191-204, RIMS, 2012. RIMS Kôkyûroku Bessatsu.

[11] A. Laurinčikas and D. Šiaučiūnas. A mixed joint universality theorem for zetafunctions. III. In A. Laurinčikas et al.(Ed.), Analytic Probab. Methods Number Theory, pp. 185-195, Vilnius, 2012. TEV.

[12] R. Macaitienè. On joint universality for zeta-functions of newforms and periodic Hurwitz zeta-functions. In K. Matsumoto et al.(Ed.), Functions in Number Theory and Their Probabilistic Aspects, volume B34, pp. 217-233, RIMS, 2012. RIMS Kôkyûroku Bessatsu.

[13] S.N. Mergelyan. Uniform approximation to functions of complex variable. Uspehi Mat. Nauk (in Russian), 7(48):31-122, 1952.

[14] H. Mishou. The joint value-distribution of the Riemann zeta-function and Hurwitz zeta-functions. Lithuaninan Mathematical Journal, 47(1):32-47, 2007. http://dx.doi.org/10.1007/s10986-007-0003-0.

[15] V. Pocevičienè and D. Šiaučiūnas. A mixed joint universality theorem for zeta-functions. II. Mathematical Modelling and Analysis, 19(1):52-65, 2014. http://dx.doi.org/10.3846/13926292.2014.893261.

[16] S.M. Voronin. Theorem on the 'universality' of the Riemann zetafunction. Mathematics of the USSR Izvestiya, 9(3):443-453, 1975. http://dx.doi.org/10.1070/IM1975v009n03ABEH001485.

[17] J.L. Walsh. Interpolation and Approximation by Rational Functions in the Complex Domain. American Mathematical Society Colloquium Publications, Vol. 20, 1968. 